\title{
DESEMPENHO DE GENÓTIPOS DE MAMOEIRO QUANTO À QUALIDADE FÍSICA E FISIOLÓGICA DE SEMENTES E ANÁLISES DE DIVERSIDADE
}

\author{
PERFORMANCE OF PAPAYA GENOTYPES RELATED THE SEEDS PHYSICAL \\ AND PHYSIOLOGICAL QUALITY AND DIVERSITY ANALYSIS
}

\section{Liana Hilda Golin MENGARDA ${ }^{1}$; José Carlos LOPES ${ }^{2}$; Rafael Fonsêca ZANOTTI ${ }^{3}$; Rodrigo Sobreira ALEXANDRE ${ }^{4}$}

1. Pós-doutoranda em Genética e Melhoramento, Centro de Ciências Agrárias, Universidade Federal do Espírito Santo - UFES, Alegre, ES, Brasil; 2. Professor, Doutor, Departamento de Produção Vegetal, Centro de Ciências Agrárias, Universidade Federal do Espírito Santo - UFES, Alegre, ES, Brasil; 3. Doutorando em Produção Vegetal, Centro de Ciências Agrárias, Universidade Federal do Espírito Santo - UFES, Alegre, ES, Brasil; 4. Professor, Doutor, Departamento de Ciências Florestais, Centro de Ciências Agrárias, Universidade Federal do Espírito Santo - UFES, Jerônimo Monteiro, ES, Brasil.

RESUMO: Objetivou-se com este trabalho avaliar o desempenho de sementes de oito genótipos de Carica papaya L. antes e após o envelhecimento acelerado. Foram avaliadas as características físicas: umidade, comprimento, diâmetro e peso de mil sementes. Sementes não envelhecidas (controle) e sementes submetidas ao envelhecimento acelerado $\left(43{ }^{\circ} \mathrm{C}\right.$ por $72 \mathrm{~h}$ ) foram semeadas em rolo de papel germitest e mantidas em BOD a 20-30 ${ }^{\circ} \mathrm{C}$. Foram avaliadas: porcentagem e frequência de germinação, índice de velocidade de germinação, tempo médio de germinação e porcentagem de plantas normais. Após 30 dias da semeadura foram avaliados comprimentos da parte aérea e da raiz, massas fresca e seca das plântulas. Em sementes não envelhecidas, o genótipo Caliman 01 apresentou maiores porcentagem, velocidade e homogeneidade de germinação, e maior porcentagem de plântulas normais. As sementes do Híbrido JS12 x Waimanalo apresentaram maior vigor após o envelhecimento ( $84 \%$ de germinação e $93 \%$ de plântulas normais). A análise de diversidade identificou como dissimilares os genótipos Caliman 01 em sementes não envelhecidas e o Híbrido JS12 x Waimanalo em sementes após o envelhecimento. Estes genótipos apresentam maior desempenho com base nas características físicas e fisiológicas das sementes e na análise de diversidade nas respectivas condições. O tempo médio de germinação é a característica que mais contribui para a separação dos genótipos em sementes não envelhecidas enquanto, em sementes envelhecidas, é o comprimento de raiz.

PALAVRAS-CHAVE: Carica papaya L. Germinação. Vigor. Teste de envelhecimento acelerado. Estatística multivariada.

\section{INTRODUÇÃO}

A cadeia produtiva do mamão representa uma importante atividade econômica para o Brasil. Algumas características da cultura oneram os custos de produção, principalmente aquelas relacionadas aos gastos com sementes. O mamoeiro apresenta um ciclo de produção com aproveitamento comercial de aproximadamente dois anos, e, ainda, deve ser feito o plantio de três mudas por cova, para garantir a permanência de pelo menos uma planta hermafrodita, triplicando o número de propágulos semeados. Desta forma, a grande demanda e o elevado preço das sementes têm levado muitos fruticultores a utilizar plantios sucessivos com as gerações F2, F3 e F4 de híbridos, causando problemas relacionados à perda de vigor $\mathrm{e}$ segregação (MARIN et al., 2006 a, b ; SERRANO; CATTANEO, 2010). Tais informações evidenciam a necessidade de pesquisas que identifiquem genótipos superiores em relação à qualidade física $\mathrm{e}$ fisiológica de sementes de mamão.

A qualidade fisiológica das sementes baseia-se num conjunto de características que determinam seu valor para a semeadura, sugerindo que o potencial de desempenho das sementes somente pode ser identificado quando considerada, além da sanidade, a interação de atributos de natureza genética, física e fisiológica (HAMPTON, 2002). Estudos que avaliam a qualidade fisiológica de sementes trazem informações importantes para a produção, armazenamento e otimização da germinação de sementes de mamão (MARTINS et al., 2005; SARI et al., 2005; TOKUHISA et al., 2007, 2008; BERBERT et al., 2008; LOPES et al., 2009; CARDOSO et al., 2009; SANTOS et al., 2009, DIAS et al., 2010). No sentido de se obter lavouras mais produtivas e frutos de ótima qualidade, soma-se a contribuição do melhoramento genético, que disponibiliza sementes com atributos aperfeiçoados e desempenho eficiente, trazendo benefícios quanto às práticas culturais, propagação e produção (MARCOS FILHO, 2005; MELETTI et al., 2011).

O teste de germinação é usado mundialmente para avaliar a qualidade fisiológica de sementes de diferentes lotes, genótipos ou espécies. No entanto, recomendam-se a utilização de 
outros testes, como aqueles que avaliam o vigor das sementes, assim como o teste de envelhecimento acelerado, considerado um dos mais sensíveis e eficientes em estimar o vigor das sementes para diversas espécies (AOSA, 2002). Este teste também é usado para identificar o potencial de desempenho de sementes de genótipos, uma vez que pode indicar materiais resistentes ou tolerantes a estresses (MARCOS FILHO, 2005). Embora as amostras apresentem germinação semelhante, podem apresentar diferentes níveis de vigor, o que é atribuído ao fato de que as primeiras alterações nos processos bioquímicos associados com a deterioração manifestam-se antes de ocorrer redução significativa na capacidade germinativa (DELOUCHE; BASKIN, 1973). O teste de envelhecimento acelerado caracteriza-se pelo uso de condições artificiais de elevada umidade e temperatura para acelerar a deterioração e identificar o vigor entre amostras estudadas. Para a sua condução de forma tradicional, Hampton e TeKrony (1995) sugerem o uso da combinação de temperatura de 41 a $43{ }^{\circ} \mathrm{C}$, pelo período de 48 a 72 h.

$\mathrm{O}$ vigor de sementes deve ser avaliado com base num conjunto de características que predizem seu potencial para uma emergência rápida e uniforme, e o desenvolvimento de plântulas normais sob diferentes condições ambientais (AOSA, 2002; HAMPTON, 2002). Para isto, além dos testes estatísticos univariados, como testes de médias, amplamente utilizados na experimentação na área de sementes, a estatística multivariada estuda fenômenos analisando simultaneamente diversas respostas, conduzindo a análises mais informativas. As análises de diversidade são eficientes para avaliar os indivíduos em vários aspectos e proporcionar uma visão holística de cada genótipo (CRUZ et al., 2011).

Desta forma, objetivou-se avaliar o desempenho de oito genótipos de mamoeiro (Carica papaya), antes e após o envelhecimento acelerado, com base na avaliação das características físicas e fisiológicas das sementes, e na análise de diversidade entre os genótipos.

\section{MATERIAL E MÉTODOS}

O trabalho foi desenvolvido no Laboratório de Análise de Sementes (LAS) do Centro de Ciências Agrárias da Universidade Federal do Espírito Santo (CCA-UFES). Foram utilizadas sementes de oito genótipos de mamoeiro (Carica papaya L.): Caliman 01 geração F2 (G1); Tainung 01 geração F2 (G2); Golden (G3); Caliman 01 (G4);
Sunrise Solo (G5); THB (G6); Híbrido Triplo (G7); e Híbrido JS12 x Waimanalo (G8). O material foi fornecido pela empresa Caliman Agrícola S/A. Foram realizados os seguintes procedimentos e avaliações:

\section{Caracterização física}

A umidade inicial das sementes (U\%) foi obtido por meio do método de estufa a $105 \pm 3{ }^{\circ} \mathrm{C}$ por $24 \mathrm{~h}$, utilizando-se três subamostras de $0,18 \mathrm{~g}$ para cada genótipo e os resultados expressos em porcentagem. $\mathrm{O}$ tamanho das sementes foi obtido com o auxílio de um paquímetro digital, sendo o comprimento (C) caracterizado como a distância entre a base e o ápice, e o diâmetro (D) a medida da porção mediana das sementes $(\mathrm{mm})$. $\mathrm{O}$ peso de mil sementes (PMS) foi calculado de acordo com Brasil (2009), atendendo os limites permitidos de coeficientes de variação (CV\%).

\section{Envelhecimento acelerado}

As sementes foram distribuídas em camada única sobre tela suspensa no interior da caixa plástica gerbox com $40 \mathrm{~mL}$ de água destilada, em câmara tipo BOD regulada à temperatura de $43{ }^{\circ} \mathrm{C}$ por $72 \mathrm{~h}$ (HAMPTON; TEKRONY, 1995; MARCOS FILHO, 2005).

\section{Germinação}

$\mathrm{O}$ teste de germinação foi conduzido em sementes não envelhecidas (Antes EA) e após o envelhecimento acelerado (EA). As sementes foram distribuídas em rolos de papel (três folhas de papel germitest), umedecidos com água destilada na proporção equivalente a 2,5 vezes a massa do papel seco, e mantidas em câmara de germinação tipo BOD regulada à temperatura alternada de $20-30$ ${ }^{\circ} \mathrm{C}$. As avaliações foram feitas diariamente, sendo calculados a germinação (G\%), o índice de velocidade de germinação (IVG) (sementes germinadas com a protrusão radicular de $0,2 \mathrm{~cm}$, até os 30 dias após a semeadura, de acordo com o modelo proposto por Maguire (1962)); o tempo médio de germinação (TMG) (LABOURIAU, 1983), sendo os resultados expressos em dias; a porcentagem de plântulas normais (PN\%), calculado em relação ao total de plântulas germinadas $(\mathrm{G} \%)$ no final do teste (BRASIL, 2009).

\section{Comprimento e massa das plântulas}

Após 30 dias da instalação do experimento foram avaliados o comprimento da parte aérea (CPA) e da raiz (CR) das plântulas normais, expressos em $\mathrm{cm}$ plântula ${ }^{-1}$, e realizada medida de massa fresca (MF) e massa seca (MS) (estufa de 
circulação forçada a $70{ }^{\circ} \mathrm{C}$ por $72 \mathrm{~h}$ ), em balança analítica $(0,0001 \mathrm{~g})$, e os resultados expressas em mg plântula ${ }^{-1}$.

\section{Delineamento e análise estatística}

$\mathrm{O}$ experimento foi conduzido num delineamento inteiramente casualizado (DIC), com quatro repetições de 25 sementes por tratamento, em esquema fatorial $2 \times 8$, sendo duas condições da semente (Antes EA e EA) e oito genótipos (G1, G2, G3, G4, G5, G6, G7 e G8). Os resultados obtidos foram submetidos à análise de variância e ao teste de Tukey, em nível de 5\% de probabilidade (ASSISTAT 7.6, 2012). A partir dos dados obtidos, foi realizada análise multivariada da divergência genética objetivando identificar a dissimilaridade entre genótipos e, assim, o seu desempenho diferencial em relação a todos os parâmetros avaliados. Para as características da qualidade física (U\%, C, D, PMS) e fisiológica (G\%, IVG, TMG, PN\%, CPA, CR, MF, MS) das sementes foi obtida a Distância Euclidiana Média Padronizada e, posteriormente, efetuados os métodos de agrupamento hierárquico de Ligação Média entre Grupo ("Unweighted Pair Group Method with Arithmetic Mean" - UPGMA). Com base nas mesmas características aplicou-se o método de otimização de agrupamento de Tocher (CRUZ; CARNEIRO, 2003), utilizando-se a distância generalizada de Mahalanobis $\left(\mathrm{D}^{2}\right)$ como medida de dissimilaridade. Utilizou-se o critério de Singh
(1981) para quantificar a contribuição relativa de cada característica para a divergência genética (CRUZ, 2006).

\section{RESULTADOS E DISCUSSÃO}

Com relação à umidade inicial das sementes, observaram-se médias entre 5,01 (G7) e 7,96 (G2) (base úmida) para os diferentes genótipos (Tabela 1). O teor de água das sementes verificado nos genótipos em estudo é adequado para a manutenção do vigor das sementes durante $\mathbf{o}$ armazenamento, visto que o comportamento das sementes de mamão se aproxima daquele observado em sementes ortodoxas, que têm sua viabilidade estendida pela redução do teor de água (BERBERT et al., 2008, DIAS et al., 2010). No entanto, a variação no teor de água pode influenciar na velocidade de umedecimento das sementes durante o tratamento de envelhecimento e determinar diferenças na intensidade de deterioração. Além disso, teores de água abaixo dos ideais ou a incapacidade de absorção adequada de água durante a embebição podem comprometer $\mathrm{o}$ processo germinativo (MARCOS FILHO, 2005). Berbert et al. (2008) verificaram que a secagem das sementes de mamão Caliman 01 para teor de água de 7,7\% tem efeito positivo sobre o seu vigor, valores próximos daqueles verificados para os genótipos G1, G2 e G4 neste trabalho.

Tabela 1. Características físicas das sementes: umidade (U), em porcentagem (\%), comprimento (C) e diâmetro (D), em milímetros (mm), e peso de mil sementes (PMS), em gramas (g), de oito genótipos de mamoeiro.

\begin{tabular}{|c|c|c|c|c|c|c|c|c|}
\hline Genótipo & \multicolumn{2}{|c|}{$\mathrm{U}(\%)$} & \multicolumn{2}{|c|}{$\mathrm{C}(\mathrm{mm})$} & \multicolumn{2}{|c|}{$\mathrm{D}(\mathrm{mm})$} & \multicolumn{2}{|c|}{ PMS (g) } \\
\hline G1 & 7,70 & $\mathrm{a}$ & 4,21 & $\mathrm{c}$ & 2,74 & $\mathrm{Bc}$ & 17,2 & A \\
\hline G2 & 7,96 & $\mathrm{a}$ & 4,22 & $\mathrm{c}$ & 2,67 & $\mathrm{C}$ & 12,7 & $\mathrm{C}$ \\
\hline G3 & 5,52 & $\mathrm{~d}$ & 3,66 & $\mathrm{~d}$ & 2,24 & $\mathrm{D}$ & 13,4 & $\mathrm{C}$ \\
\hline G4 & 7,04 & $a b$ & 5,52 & $\mathrm{a}$ & 3,05 & $\mathrm{Ab}$ & 17,9 & A \\
\hline G5 & 6,57 & $\mathrm{bc}$ & 4,58 & $\mathrm{bc}$ & 3,01 & $a b c$ & 14,2 & $\mathrm{C}$ \\
\hline G6 & 5,97 & $\mathrm{~cd}$ & 4,48 & $\mathrm{bc}$ & 3,07 & $\mathrm{Ab}$ & 13,4 & $\mathrm{C}$ \\
\hline G7 & 5,01 & $\mathrm{~d}$ & 5,00 & $a b$ & 2,87 & $a b c$ & 15,3 & $\mathrm{~B}$ \\
\hline G8 & 5,85 & $\mathrm{~cd}$ & 5,18 & $\mathrm{a}$ & 3,16 & $\mathrm{~A}$ & 13,7 & $\mathrm{C}$ \\
\hline $\mathbf{F}$ & \multicolumn{2}{|c|}{$33,80 * *$} & \multicolumn{2}{|c|}{$25.90 * *$} & \multicolumn{2}{|c|}{$16.23 * *$} & \multicolumn{2}{|c|}{$35.74 * *$} \\
\hline CV $(\%)$ & \multicolumn{2}{|c|}{5,02} & \multicolumn{2}{|c|}{5,15} & \multicolumn{2}{|c|}{5.16} & \multicolumn{2}{|c|}{7,63} \\
\hline
\end{tabular}

Médias seguidas de letras iguais (colunas) não diferem entre si pelo teste de Tukey em nível de $1 \%$ de probabilidade $(\mathrm{n}=4)$ ). $\mathrm{F}=$ valor de F calculado obtido pela análise de variância. CV $(\%)=$ coeficiente de variação.

Os genótipos G4, G7 e G8 apresentaram maior comprimento e diâmetro médio, não diferindo dos genótipos G5 e G6. Os genótipos G1 e G4 apresentaram maior peso de mil sementes (PMS). Segundo Martins et al. (2005), sementes de mamão de maior peso (PMS entre 17 e $19 \mathrm{~g}$ ), apresentam melhor resposta quanto à avaliação do vigor, valores correspondentes aos verificados neste trabalho para G1 (17,2 g) e G4 (17,9 g).

De acordo com o teste de germinação de sementes não envelhecidas (Antes EA), o genótipo Caliman 01 (G4) apresentou maior porcentagem de 
germinação (94\%) maior IVG $(2,30)$, e menor tempo médio de germinação (9 dias) comparado aos demais genótipos (Tabela 2). Também apresentou alta porcentagem de plântulas normais (98\%). Estudos anatômicos com sementes de mamão permitiram observar a existência de diferenças estruturais e dimensionais entre diferentes genótipos, o que pode interferir diretamente nas respostas às condições de germinação a que são expostas as sementes (SANTOS et al., 2009). No presente estudo, os fatores mais evidentes com relação à diferenciação dos lotes parecem estar relacionados às características genéticas, fator que atua em potencial na qualidade fisiológica e no vigor das sementes (CARDOSO et al., 2009).

Tabela 2. Porcentagem de germinação (G), em porcentagem (\%), índice de velocidade de germinação (IVG), tempo médio de germinação (TMG), em dias, e porcentagem de plântulas normais (PN), em porcentagem (\%), de sementes não envelhecidas (Antes EA) e após o envelhecimento acelerado (EA) $\left(43^{\circ} \mathrm{C}\right.$ por $\left.72 \mathrm{~h}\right)$, de oito genótipos de mamoeiro.

\begin{tabular}{|c|c|c|c|c|c|c|c|c|c|c|c|c|c|c|c|c|}
\hline \multirow{2}{*}{ Genótipo } & \multicolumn{4}{|c|}{ G(\%) } & \multicolumn{4}{|c|}{ IVG } & \multicolumn{4}{|c|}{ TMG (dias) } & \multicolumn{4}{|c|}{ PN (\%) } \\
\hline & \multicolumn{2}{|c|}{ Antes EA } & \multicolumn{2}{|c|}{ EA } & \multicolumn{2}{|c|}{ Antes EA } & \multicolumn{2}{|c|}{ EA } & \multicolumn{2}{|c|}{ Antes EA } & \multicolumn{2}{|c|}{ EA } & \multicolumn{2}{|c|}{ Antes EA } & \multicolumn{2}{|c|}{ EA } \\
\hline G1 & 73 & $\mathrm{bcA}$ & 59 & $\mathrm{bB}$ & 1,4 & $\mathrm{bcA}$ & 1,6 & $\mathrm{bcA}$ & 15 & $\mathrm{aA}$ & 10 & $\mathrm{~dB}$ & 82 & abcA & 76 & $\mathrm{bcB}$ \\
\hline G2 & 59 & $\mathrm{cA}$ & 48 & $\mathrm{bB}$ & 1,2 & $\mathrm{cB}$ & 1,5 & $\mathrm{bcA}$ & 16 & $\mathrm{aA}$ & 13 & $a b c B$ & 75 & $\mathrm{bcA}$ & 65 & $\mathrm{cA}$ \\
\hline G3 & 65 & $\mathrm{bcA}$ & 77 & $\mathrm{bA}$ & 1,1 & $\mathrm{cB}$ & 1,8 & $\mathrm{bcA}$ & 15 & $\mathrm{abA}$ & 11 & $\mathrm{cdB}$ & 92 & $\mathrm{abA}$ & 68 & $\mathrm{cB}$ \\
\hline G4 & 94 & $\mathrm{aA}$ & 59 & $\mathrm{bB}$ & 2,3 & $\mathrm{aA}$ & 2,3 & $\mathrm{aA}$ & 09 & $\mathrm{dA}$ & 10 & $\mathrm{dA}$ & 98 & $\mathrm{aA}$ & 78 & $b c B$ \\
\hline G5 & 62 & $\mathrm{bcA}$ & 65 & $\mathrm{bA}$ & 1,4 & $b c B$ & 1,9 & bA & 13 & $\mathrm{bcA}$ & 12 & $\operatorname{cdA}$ & 73 & bB & 100 & $\mathrm{aA}$ \\
\hline G6 & 64 & $\mathrm{bcA}$ & 59 & $\mathrm{bA}$ & 1,2 & $c B$ & 1,4 & $\mathrm{cA}$ & 15 & $\mathrm{aA}$ & 14 & $\mathrm{abA}$ & 87 & $a b c A$ & 77 & bcA \\
\hline G7 & 64 & bcA & 56 & $\mathrm{bA}$ & 1,2 & $\mathrm{cA}$ & 1,1 & $\mathrm{dA}$ & 10 & $\mathrm{~dB}$ & 15 & $\mathrm{aA}$ & 85 & $a b c A$ & 80 & bcA \\
\hline G8 & 77 & $\mathrm{bB}$ & 84 & $\mathrm{aA}$ & 1,8 & $\mathrm{bA}$ & 1,9 & $\mathrm{bA}$ & 11 & $\operatorname{cdA}$ & 13 & $\mathrm{bcA}$ & 84 & $a b c A$ & 93 & $\mathrm{abA}$ \\
\hline $\mathbf{F}$ & \multicolumn{4}{|c|}{$9,14 * *$} & \multicolumn{4}{|c|}{$4,90 * *$} & \multicolumn{4}{|c|}{$19,05^{* *}$} & \multicolumn{4}{|c|}{$7,96 * *$} \\
\hline $\mathrm{CV}(\%)$ & \multicolumn{4}{|c|}{7,99} & \multicolumn{4}{|c|}{4,01} & \multicolumn{4}{|c|}{7,99} & \multicolumn{4}{|c|}{9,99} \\
\hline
\end{tabular}

Médias seguidas de letras iguais, minúsculas nas colunas e maiúsculas nas linhas (entre as colunas Antes EA e EA), não diferem entre si pelo teste de Tukey em nível de $1 \%$ de probabilidade $(\mathrm{n}=4) . \mathrm{F}=$ valor de $\mathrm{F}$ calculado obtido pela análise de variância. $\mathrm{CV}(\%)=$ coeficiente de variação.

Nas sementes submetidas ao envelhecimento acelerado (EA), o genótipo Híbrido JS12 x Waimanalo (G8) apresentou maior germinação $(84 \%)$, quando comparado às sementes não envelhecidas (C) (77\%) (Tabela 2). Isto chama atenção, pois, de um modo geral, as sementes dos demais genótipos apresentaram redução ou mantiveram os valores de germinação estatisticamente semelhantes após o envelhecimento.

O teste de envelhecimento acelerado, embora tenha sido desenvolvido com o objetivo de identificar o potencial de armazenamento de sementes (DELOUCHE; BASKIN, 1973), também tem sido usado para identificar o potencial de desempenho de sementes, uma vez que pode indicar materiais resistentes ou tolerantes a estresses (MARCOS FILHO, 2005). Acredita-se, portanto, que o G8 apresente características germinativas singulares que podem estar relacionadas a questões de resistência a estresses: as suas sementes expressam maior vigor de germinação após serem submetidas ao estresse de temperatura e umidade elevadas.

As respostas observadas nos diferentes genótipos podem estar relacionadas também ao fenômeno de superação da dormência. A intensa domesticação e os trabalhos de melhoramento genético vegetal procuram promover a remoção dos mecanismos de dormência das sementes, sendo esta principalmente determinada por fatores genéticos, com certa plasticidade na expressão genotípica pela influência do ambiente. Assim, sob condições ambientais menos favoráveis a dormência pode se manifestar, mesmo em espécies cultivadas (MARCOS FILHO, 2005), como o mamoeiro. A dormência é comumente observada em sementes de C. papaya (SARI et al., 2005; TOKUHISA et al., 2007, 2008, LOPES et al., 2009; DIAS et al., 2010). Trabalho realizado por Tokuhisa et al. (2007) utilizaram o EA como método de superação de dormência de sementes de C. papaya Tainung 01 .

Após o envelhecimento, os genótipos G5 e G8 apresentaram aumento da porcentagem de plântulas normais (de 73 para $100 \%$; de 84 para 93\%, respectivamente), sem diferir estatisticamente dos genótipos G1, G2, G6 e G7 (Tabela 2). O G8, além de apresentar maior potencial germinativo após suas sementes serem submetidas ao EA, apresentou redução da formação de plântulas anormais, sugerindo superação de dormência e/ou resistência ao estresse. Os genótipos G1, G2 e G4 
apresentaram redução significativa da porcentagem de germinação após o EA, e os genótipos G3 e G4 apresentaram redução na porcentagem de plântulas normais após o EA, indicando que a condição de envelhecimento $\left(43^{\circ} \mathrm{C}\right.$ por $\left.72 \mathrm{~h}\right)$ pode ter acelerado a deterioração das sementes destes genótipos.

O tempo médio de germinação das sementes variou de 09 a 16 dias. Observa-se que em cinco dos oito genótipos estudados, as sementes que foram submetidas ao EA apresentaram redução do tempo médio de germinação (Tabela 2). $\mathrm{O}$ genótipo Caliman 01 (G4) apresentou o menor tempo médio de germinação (9 dias) associado a maior média de porcentagem de plântulas normais nas sementes não envelhecidas.

Com relação ao desenvolvimento das plântulas (Tabela 3), em sementes não envelhecidas (Antes EA), maiores CPA $(6,2 \mathrm{~cm})$ e CR $(3,4 \mathrm{~cm})$ foram observados para o G6, maior média de massa fresca (MF) (99 mg) foi verificado no G4 e maior média de massa seca (MS) $(5,4 \mathrm{mg})$ no G1, diferindo estatisticamente dos demais genótipos avaliados.

Tabela 3. Comprimento de parte aérea $(\mathrm{CPA})$ e comprimento da raiz $(\mathrm{CR})$, em centímetro $(\mathrm{cm})$, massa fresca (MF) e massa seca (MS), em miligramas (mg), de plântulas após 30 dias da semeadura, provenientes de sementes não envelhecidas (Antes EA) e após o envelhecimento acelerado (EA) $\left(43^{\circ} \mathrm{C}\right.$ por $\left.72 \mathrm{~h}\right)$, de oito genótipos de mamoeiro.

\begin{tabular}{|c|c|c|c|c|c|c|c|c|c|c|c|c|c|c|c|c|}
\hline \multirow{3}{*}{$\begin{array}{c}\text { Genótipo } \\
\text { G1 }\end{array}$} & \multicolumn{4}{|c|}{ CPA (cm) } & \multicolumn{4}{|c|}{$\mathrm{CR}(\mathrm{cm})$} & \multicolumn{4}{|c|}{ MF (mg) } & \multicolumn{4}{|c|}{ MS (mg) } \\
\hline & \multicolumn{2}{|c|}{ Antes EA } & \multicolumn{2}{|c|}{ EA } & \multicolumn{2}{|c|}{ Antes EA } & \multicolumn{2}{|c|}{ EA } & \multicolumn{2}{|c|}{ Antes EA } & \multicolumn{2}{|c|}{ EA } & \multicolumn{2}{|c|}{ Antes EA } & \multicolumn{2}{|c|}{ EA } \\
\hline & 2,8 & $\mathrm{cA}$ & 3,0 & $\mathrm{dA}$ & 0,7 & $\mathrm{dA}$ & 0,9 & $\mathrm{cA}$ & 61 & bA & 52 & $\mathrm{bA}$ & 5,4 & $\mathrm{aA}$ & 3,5 & $\mathrm{aB}$ \\
\hline G2 & 2,9 & $\mathrm{cA}$ & 3,4 & $\operatorname{cdA}$ & 0,5 & $\mathrm{~dB}$ & 0,9 & $\mathrm{cA}$ & 45 & $\mathrm{cA}$ & 52 & BA & 4,3 & $\mathrm{bA}$ & 2,9 & $\mathrm{aB}$ \\
\hline G3 & 4,9 & bA & 3,2 & $\mathrm{~dB}$ & 2,0 & bA & 1,9 & bA & 58 & bcA & 58 & bA & 3,5 & $\operatorname{cdA}$ & 3,3 & $\mathrm{aA}$ \\
\hline G4 & 4,8 & bA & 4,4 & bcA & 1,6 & bA & 1,6 & bA & 99 & $\mathrm{aA}$ & 48 & bB & 2,5 & $\mathrm{dA}$ & 2,9 & $\mathrm{aA}$ \\
\hline G5 & 4,7 & bA & 4,5 & bcA & 1,5 & $\mathrm{bcA}$ & 1,4 & $\mathrm{bcA}$ & 60 & bcA & 62 & bA & 4,1 & $\mathrm{bcA}$ & 3,3 & $\mathrm{aB}$ \\
\hline G6 & 6,2 & $\mathrm{aA}$ & 4,6 & bB & 3,4 & $\mathrm{aA}$ & 1,5 & bB & 70 & $\mathrm{bA}$ & 48 & bB & 3,6 & $\mathrm{bcA}$ & 3,0 & $\mathrm{aB}$ \\
\hline G7 & 4,8 & bA & 4,0 & bcdB & 1,5 & $\mathrm{bcA}$ & 1,5 & bA & 55 & bcA & 56 & bA & 3,5 & $\operatorname{cdA}$ & 3,7 & $\mathrm{aA}$ \\
\hline G8 & 4,4 & $\mathrm{bB}$ & 6,0 & $\mathrm{aA}$ & 1,1 & $\mathrm{cdB}$ & 4,0 & $\mathrm{aA}$ & 57 & $\mathrm{bcB}$ & 82 & $\mathrm{aA}$ & 3,1 & $\operatorname{cdA}$ & 3,5 & $\mathrm{aA}$ \\
\hline $\mathbf{F}$ & \multicolumn{4}{|c|}{$9,46 * *$} & \multicolumn{4}{|c|}{$61,54 * *$} & \multicolumn{4}{|c|}{$19,96 * *$} & \multicolumn{4}{|c|}{$7,13 * *$} \\
\hline CV (\%) & \multicolumn{4}{|c|}{11,87} & \multicolumn{4}{|c|}{14,61} & \multicolumn{4}{|c|}{11,87} & \multicolumn{4}{|c|}{12,86} \\
\hline
\end{tabular}

Médias seguidas de letras iguais, minúsculas nas colunas e maiúsculas nas linhas (entre as colunas Antes EA e EA), não diferem entre si pelo teste de Tukey em nível de $1 \%$ de probabilidade $(\mathrm{n}=4)$. $\mathrm{F}=$ valor de $\mathrm{F}$ calculado obtido pela análise de variância. $\mathrm{CV}(\%)=$ coeficiente de variação.

O EA promoveu a redução ou manutenção dos valores médios de comprimento da parte aérea (CPA), à exceção do G8, que apresentou maior CPA após o envelhecimento (Tabela 3); para o comprimento da raiz (CR) além do G8, também o genótipo G2 apresentou maior média de CR. A exposição ao estresse do envelhecimento acelerado, ou seja, à temperatura e umidade relativa elevadas, pode determinar a ativação de certas enzimas, que podem influenciar nas atividades metabólicas e desta forma, promover um maior crescimento, conforme verificado pelo aumento do comprimento do hipocótilo e da raiz primária (BINOTTI et al., 2008). Os genótipos (G2 e G8), que apresentaram maior CPA e CR após o EA podem, portanto, terem sofrido influência positiva da temperatura na ativação destas enzimas.

Com relação à massa fresca das plântulas, somente o genótipo G8 apresentou médias superiores após EA, quando comparada às sementes não envelhecidas (Antes EA). Não foi verificada variação significativa quanto ao valor de MS entre os genótipos na condição de EA.

Entre as manifestações fisiológicas da redução do vigor de sementes estão a redução da velocidade de germinação e de crescimento, redução da porcentagem de germinação e o aumento da taxa de anormalidade de plântulas. Logo, a qualidade fisiológica das sementes deve levar em conta a formação de plântulas normais e vigorosas (AOSA, 2002; MARCOS FILHO, 2005), e a avaliação conjunta de G\%, IVG, TMG, associada aos parâmetros de desenvolvimento das plântulas, permite inferir que o genótipo G4 apresenta maior vigor de sementes não envelhecidas (Antes EA), e o G8 apresenta maior vigor de sementes após o envelhecimento acelerado.

Os métodos de agrupamento UPGMA e de otimização de agrupamento de Tocher representam técnicas eficientes para avaliar os indivíduos em vários aspectos e proporcionar uma visão holística de cada genótipo (CRUZ et al., 2011). 
Desempenho de genótipos...

Após a separação dos grupos por meio da análise de agrupamento UPGMA, com base nas 12 características de qualidade física e fisiológica das sementes não envelhecidas (Antes EA), os genótipos foram separados em dois grupos, estabelecendo ponto de corte a $70 \%$ do nível máximo de fusão, sendo o genótipo Caliman 01 (G4) representado em um grupo isolado (Figura 1A). Este apresentou médias de $\mathrm{PMS}=17,9, \mathrm{G}=$
MENGARDA, L. H. G. et al.

$94 \%, \mathrm{IVG}=2,3, \mathrm{PN}=98 \%, \mathrm{CPA}=4,8, \mathrm{CR}=1,6, \mathrm{e}$ $\mathrm{MF}=99$. O outro grupo, formado pelos demais genótipos, apresentou médias de $\mathrm{PMS}=14,3, \mathrm{G}=$ $66,3 \%, \mathrm{IVG}=1,3, \mathrm{PN}=82,7 \%, \mathrm{CPA}=4,39, \mathrm{CR}=$ 1,53 , e $\mathrm{MF}=58$, e $\mathrm{MS}=3,93$. Logo, o genótipo Caliman 01 apresenta médias superiores que as médias dos demais genótipos para a grande maioria das características avaliadas.

SEMENTES NĀO ENVELHECWAS

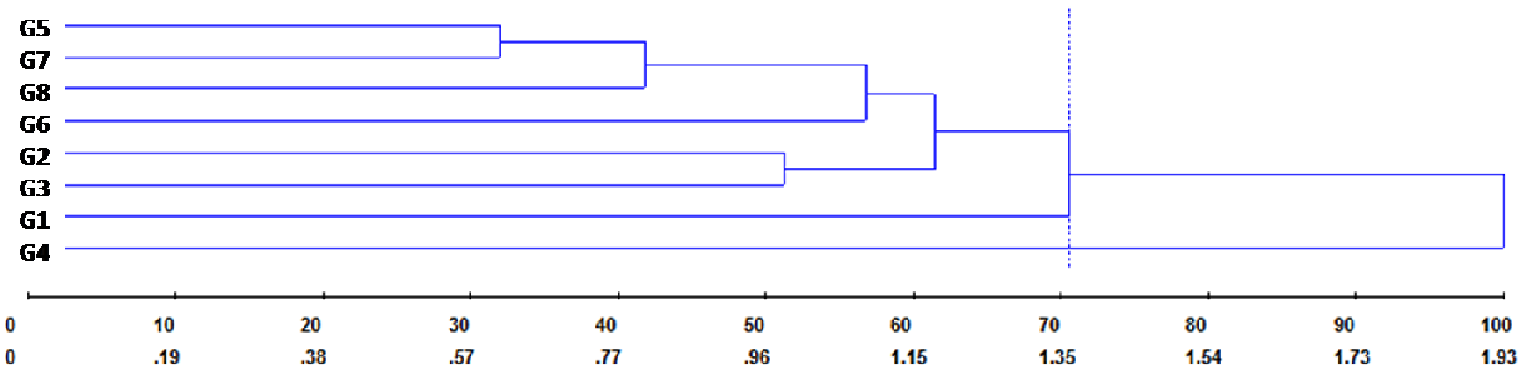

AIYS K,NV KIIIKCIMKNTO ACWI,KRAIM)

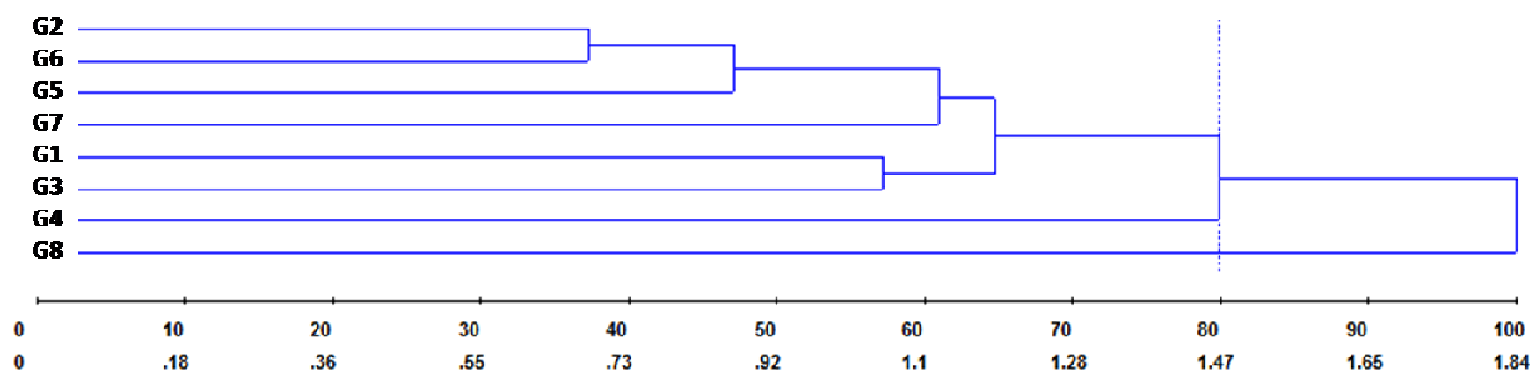

Figura 1. Dendrograma UPGMA para dados físicos e fisiológicos de sementes de oito genótipos de mamoeiro. A - sementes não envelhecidas; B - sementes após o envelhecimento acelerado ( $43{ }^{\circ} \mathrm{C}$ por 72 h).

Nas sementes envelhecidas (EA), os genótipos também foram divididos em dois grupos (ponto de corte a $80 \%$ do nível máximo de fusão), sendo que o genótipo JS12 x Waimanalo (G8) apresentou-se num grupo isolado (Figura 1B), com médias de $\mathrm{C}=5,18, \mathrm{D}=3,16, \mathrm{G}=84 \%, \mathrm{IVG}=1,9$, $\mathrm{PN}=93 \%, \mathrm{CPA}=6,0, \mathrm{CR}=4,0, \mathrm{MF}=82$ e $\mathrm{MS}=$ 3,5 . O outro grupo, formado pelos demais genótipos, apresentou médias de $\mathrm{C}=4,52, \mathrm{D}=2,82$, $\mathrm{G}=60,4 \%, \mathrm{IVG}=1,7, \mathrm{PN}=77,7 \%, \mathrm{CPA}=3,87$, $\mathrm{CR}=1,39, \mathrm{MF}=53,7$ e $\mathrm{MS}=3,23$. Assim, $\mathrm{o}$ genótipo JS12 x Waimanalo apresenta médias superiores que as médias dos demais genótipos para a grande maioria das características avaliadas após o envelhecimento.

Desta forma, dados relacionados a aspectos agronômicos, como a qualidade física e fisiológica de sementes, avaliados quanto à divergência ou similaridade permite separar genótipos com sementes de desempenho superior e inferior. Com base em análises multivariadas, Cardoso et al. (2009), trabalhando com 30 genótipos de mamão constataram elevada divergência para atributos relacionados à qualidade fisiológica de sementes entre os diferentes genótipos, separando-os com base em seu desempenho germinativo.

Utilizando-se o método de otimização de agrupamento de Tocher (Tabela 4), para sementes não envelhecidas (Antes EA) os genótipos foram separados em quatro grupos, sendo o genótipo Caliman 01 (G4) novamente sendo representado em um grupo isolado. As médias do grupo 4, representado por este genótipo, foram superiores as médias dos demais grupos para as características 
$\mathrm{PMS}=17,9, \mathrm{G}=94 \%, \mathrm{IVG}=2,3, \mathrm{PN}=98 \%$, e MF $=99$. O grupo 1, representado pelos genótipos Sunrise Solo (G5), Híbrido Triplo (G7) e Híbrido JS12 x Waimanalo (G8) apresentou as seguinte médias: $\mathrm{PMS}=14,4, \mathrm{G}=67,7 \%, \mathrm{IVG}=1,47, \mathrm{PN}=$ $80,7 \%$, e MF $=57,3$. O grupo 2, representado pelos genótipos Caliman 01 geração F2 (G1); Tainung 01 geração $\mathrm{F} 2(\mathrm{G} 2)$ : $\mathrm{PMS}=15, \mathrm{G}=66 \%, \mathrm{IVG}=1,3$, $\mathrm{PN}=78,5 \%$, e $\mathrm{MF}=53$. E o grupo 3, representado pelos genótipos Golden (G3) e THB (G6): PMS = $13,4, \mathrm{G}=64,5 \%, \mathrm{IVG}=1,15, \mathrm{PN}=89,5 \%, \mathrm{e} \mathrm{MF}=$ 64.

Tabela 4. Agrupamento baseado em caracteres morfológicos, pelo método de Tocher, de sementes não envelhecidas (Antes EA) e após o envelhecimento acelerado (EA) $\left(43{ }^{\circ} \mathrm{C}\right.$ por $\left.72 \mathrm{~h}\right)$, de oito genótipos de mamoeiro.

\begin{tabular}{ccc}
\hline \multicolumn{3}{c}{ Antes EA } \\
\hline Grupo & Sub-grupo & Genótipos \\
\hline 1 & - & G5 G8 G7 \\
2 & - & G1 G2 \\
3 & - & G3 G6 \\
4 & - & G4 \\
\hline \multicolumn{3}{c}{ EA } \\
\hline Grupo & Sub-grupo & Genótipos \\
\hline 1 & 1.1 & G5 G6 G7 \\
& 1.2 & G1 G2 \\
& 1.3 & G4 \\
& 1.4 & G3 \\
2 & - & G8
\end{tabular}

Para as características das sementes envelhecidas (EA), os genótipos foram agrupados em dois grupos (Tabela 4), sendo que novamente o G8 (JS12 x Waimanalo) apresentou-se isolado dos demais genótipos, apresentando médias superiores às médias dos demais grupos para a grande maioria das características avaliadas (as médias entre os grupos 2 e 1 são as mesmas listadas anteriormente, na descrição do método UPGMA).
As características físicas e fisiológicas das sementes foram usadas para as análises de diversidade, que separaram em grupos isolados os genótipos G4 para sementes não envelhecidas, e G8 para sementes envelhecidas. Para tanto, cada característica apresenta uma contribuição relativa para a formação dos grupos (Tabela 5).

Tabela 5. Contribuição relativa $(\mathrm{Cr} \%)$ das características físicas (U\%, C, D, PMS) e fisiológicas (G\%, IVG, TMG, PN\%, MF, MS, CR, CPA) de sementes não envelhecidas (Antes EA) e após o envelhecimento acelerado (EA) $\left(43^{\circ} \mathrm{C}\right.$ por $\left.72 \mathrm{~h}\right)$, para a dissimilaridade genética de oito genótipos de mamoeiro pelo método proposto por Singh (1981), baseado em $\mathrm{D}^{2}$ de Mahalanobis (S.j).

\begin{tabular}{ccccc}
\hline & \multicolumn{2}{c}{ Antes EA } & \multicolumn{2}{c}{ EA } \\
\hline Característica & S.j & Cr\% & S.j & Cr\% \\
\hline U\% & 1040.436 & 16.1927 & 2356.847 & 30.530 \\
C & 1168.246 & 18.1818 & 317.1577 & 4.1084 \\
D & 109.7266 & 1.70770 & 320.5556 & 4.1524 \\
PMS & 99.34296 & 1.54610 & 36.13977 & 0.4681 \\
G\% & 104.7637 & 1.63050 & 39.34895 & 0.5097 \\
IVG & 502.5629 & 7.82160 & 159.6451 & 2.0680 \\
TMG & 1435.090 & 22.3348 & 0.438054 & 0.0057 \\
PN\% & 77.79553 & 1.21080 & 126.3575 & 1.6368 \\
CPA & 371.9425 & 5.78870 & 153.6967 & 1.9909 \\
CR & 700.5241 & 10.9025 & 3586.503 & 46.459
\end{tabular}




\begin{tabular}{lllll} 
MF & 224.6176 & 3.49580 & 230.4287 & 2.9849 \\
MS & 203.6011 & 3.16870 & 84.49828 & 1.0946 \\
\hline
\end{tabular}

Em sementes não envelhecidas o TMG foi a característica de maior contribuição relativa, seguida de comprimento (C), umidade (U\%) e comprimento da raiz (CR). O índice de velocidade e o tempo médio de germinação trazem informações importantes para a formação de um estande ideal de plantas (AOSA, 2002; MARCOS FILHO, 2005). Logo, a elevada contribuição relativa da característica TMG na separação dos grupos, sugere que o maior desempenho de sementes não envelhecidas tem relação direta com uma germinação mais rápida e uniforme. Cabe ressaltar que, antes que a velocidade de germinação seja alterada, eventos metabólicos já se encontram muito modificados, alterando a eficiência na síntese de compostos essenciais à germinação (MARCOS FILHO, 2005), refletindo, assim, nos demais parâmetros avaliados.

Cardoso et al. (2009) utilizaram o método de Singh para avaliar a importância relativa das oito variáveis utilizadas em análise de diversidade em mamoeiro, verificando que as variáveis que mais contribuíram para a divergência foram massa de mil sementes $(37,07 \%)$ e comprimento da raiz $(17,76 \%)$, e a característica que menos contribuiu foi a emergência $(1,48 \%)$.

$\mathrm{Na}$ formação dos grupos das sementes envelhecidas, a característica de maior contribuição relativa foi o comprimento de raiz (CR), seguido da umidade das sementes. Similarmente, outros autores relataram ser o comprimento da raiz mais sensível para diferenciar vigor de sementes como verificado em sementes de trigo (MAIA et al., 2007) e milho (VENÂNCIO et al., 2012), e separar lotes de sementes, conforme observado em lotes de sementes de alface (NASCIMENTO; PEREIRA, 2007) e de soja (VANZOLINI et al., 2007).

Durante o teste de envelhecimento acelerado, a inclusão de avaliações do crescimento das plântulas e/ou sua velocidade pode contribuir para aprimorar a qualidade das informações obtidas, diferente das avaliações inicialmente propostas para o teste, que contavam apenas com a porcentagem de plântulas normais formadas e sobreviventes (MARCOS FILHO, 2005). A alta contribuição relativa desta característica revela a importância da sua análise na avaliação do desempenho de sementes de diferentes materiais genéticos.

As análises da diversidade genética permitem identificar e selecionar genitores com características interessantes ao melhoramento genético (CRUZ et al., 2011). Neste caso, os testes de médias (univariados), realizados para cada característica separadamente, indicam que as sementes do G4 na condição de não envelhecidas (Antes EA), e do G8 para sementes envelhecidas (EA) apresentaram maiores médias para inúmeras características, o que refletiu na separação destes genótipos em grupos isolados baseado nas análises multivariadas. Assim, cada um destes genótipos apresenta características germinativas, que indicam maior desempenho das sementes em determinada condição.

Para determinar a influência das características introduzidas em novos cultivares sobre os processos fisiológicos ou vitais, deve ser conhecido o desempenho de sementes dos diferentes genótipos quanto à germinação e ao vigor. Logo, estudos como este trazem informações relevantes do ponto de vista da propagação de mamoeiro, em virtude da escassez de referências sobre a variabilidade dos caracteres relacionados à germinação e ao vigor, além do agrupamento de materiais genéticos similares. Tais informações podem subsidiar a escolha de progenitores visando à obtenção de genótipos de maior desempenho germinativo.

\section{CONCLUSÕES}

Em sementes não envelhecidas, o genótipo Caliman 01 apresenta maiores peso de mil sementes, porcentagens de germinação e de plântulas normais, velocidade e homogeneidade de germinação. Nesta condição, o genótipo Caliman 01 forma um grupo isolado, com médias superiores aos demais genótipos, sendo que o tempo médio de germinação é a característica que mais contribui para o agrupamento.

As sementes do genótipo Híbrido JS12 x Waimanalo externam maior vigor quanto submetidas ao envelhecimento acelerado. Nesta condição, o genótipo Híbrido JS12 x Waimanalo forma um grupo isolado, com médias superiores aos demais genótipos, sendo que o comprimento de raiz é a característica que mais contribui para o agrupamento.

Os genótipos Caliman 01 e Hibrido JS12 x Waimanalo apresentam maior desempenho quanto às características germinativas de sementes não envelhecidas e envelhecidas, respectivamente. 


\section{AGRADECIMENTOS}

À Caliman Agrícola S/A pelo fornecimento do material vegetal. A CAPES e ao CNPq pela concessão de bolsas de pós-graduação e de produtividade aos autores.

\begin{abstract}
The objective of this study was to evaluate the seeds performance in eight Carica papaya genotypes, before and after accelerated aging test. We evaluated the seeds physical characteristics: humidity, length, diameter and weight of thousand seeds. Not aging seeds (control) and seeds subjected to accelerated aging test $\left(43^{\circ} \mathrm{C}\right.$ for $72 \mathrm{~h}$ ) were germinates in paper roll germitest and maintained in BOD at $20-30^{\circ} \mathrm{C}$. From the daily count, was evaluated the germination percentage and frequency, the speed of germination index, germination time, and the normal plants percentage. After 30 days of culture were evaluated lengths of shoot and root, fresh and dry weight of seedlings. In control seeds, the Caliman 01 genotype had higher percentage, speed and uniformity of germination, and higher normal seedlings percentage. Seeds of the Hybrid JS12 x Waimanalo genotype showed higher vigor after aging (84\% of germination and 93\% of normal seedlings). The diversity analysis identified as dissimilar the Caliman 01 genotype in control seeds and the Hybrid JS12 x Waimanalo genotype in after aging seeds. This genotypes have higher performance based on assessments of physical and physiological quality of seeds and diversity analysis in this conditions. The germination time was the feature that most contributes to the separation of genotypes in control seeds, and the root length in after aging seeds.
\end{abstract}

KEYWORDS: Carica papaya L. Germination. Vigor. Accelerated aging test. Multivariate statistical.

\title{
REFERÊNCIAS
}

ASSISTAT 7.6 BETA. Assistat assistência estatística. Campina Grande: DEAG-CTRN-UFCG, 2012.

ASSOCIATION OF OFFICIAL SEED ANALYSTS. Seed vigor testing handbook. Lincoln: AOSA, 2002. 105p. Contribution, 32.

BERBERT, P. A.; CARLESSO, V. A.; SILVA, R. F.; ARAÚJO, E. F.; THIÉBAUT, J. T. L.; OLIVEIRA, M. T. R. Qualidade fisiológica de semente de mamão em função da secagem e do armazenamento. Revista Brasileira de Sementes, Londrina, v. 30, n. 1, p. 40-48, 2008.

BINOTTI, F. F. S.; HAGA, K. I.; CARDOSO, E. D.; ALVEZ, C. Z.; SÁ, M. E.; ARF, O. Efeito do período de envelhecimento acelerado no teste de condutividade elétrica e na qualidade fisiológica de sementes de feijão. Acta Scientiarum: Agronomy, Maringá, v. 30, n. 2, p. 247-254, 2008.

BRASIL. Ministério da Agricultura, Pecuária e Abastecimento. Regras para análise de sementes. Brasília: MAPA/DAS/ACS, 2009. 395p.

CARDOSO, D. L.; SILVA, R. F.; PEREIRA, M. G.; VIANA, A. P. Diversidade genética e parâmetros genéticos relacionados à qualidade fisiológica de sementes em germoplasma de mamoeiro. Revista Ceres, Viçosa, v. 56, n. 5, p. 572-579, 2009.

CRUZ, Cosme Damião. Programa GENES - Aplicativo Computacional em Estatística e Genética. Viçosa: UFV, 2006.

CRUZ, Cosme Damião; CARNEIRO, Pedro Crescencio Souza. Modelos Biométricos Aplicados ao Melhoramento Genético. Viçosa: UFV, 2003. 585 p.

CRUZ, Cosme Damião; FERREIRA, Fábio Medeiros; PESSONI, Luiz Alberto. Biometria aplicada ao estudo da diversidade genética. Visconde do Rio Branco: Suprema, 2011, 620p.

DELOUCHE, J. C.; BASKIN, C. C. Accelerated aging techniques for predicting the relative storability of seed lots. Seed Science and Technology, Wageningen, v. 1, n. 2, p. 427-452, 1973. 
DIAS, D. C. F. S.; ESTANISLAU, W. T.; FINGER, F. L.; DIAS, L. A. S. Physiological and enzymatic alterations in papaya seed during storage. Revista Brasileira de Sementes, Londrina, v. 32, n. 1 p. 148-157, 2010.

HAMPTON, J. G. What is seed quality? Seed Science and Technology, Zürich, v. 30, n. 1, p. 1-10, 2002.

HAMPTON, John G.; TeKRONY, Dennis M. Handbook of vigour test methods. Zürich: ISTA. 1995. 117p.

LABOURIAU, Luiz Fernando Gouvea. G. A germinação das sementes. Washington: Secretaria Geral da Organização dos Estados Americanos, 1983. 174 p.

LOPES, A. W. P.; SELEGUINI, A.; BOLIANI, A. C.; CÔRREA, L. S. Estádio de maturação do fruto e uso do ácido giberélico na germinação de sementes de mamoeiro. Pesquisa Agropecuária Tropical, Goiânia, v. 39, n. 4, p. 278-284, 2009.

MAIA, A. R.; LOPES, J.C; TEIXEIRA, C. O. Efeito do envelhecimento acelerado na avaliação da qualidade fisiológica de sementes de trigo. Ciência e Agrotecnologia, Lavras, v. 31, n. 3, p. 678-684, 2007.

MAGUIRE, J. D. Speeds of germination-aid selection and evaluation for seedling emergence and vigor. Crop Science, Madison, v.2, p.176-177, 1962. http://dx.doi.org/10.2135/cropsci1962.0011183X000200020033x

MARCOS FILHO, Julio. Fisiologia de sementes de plantas cultivadas. Piracicaba: FEALQ, 2005. 495p.

MARIN, S. L. D.; PEREIRA, M. G.; AMARAL JR., A. T.; MARTELLETO, L. A. P.; IDE, C. D. Heterosis in papaya hybrids from partial diallel of 'Solo' and 'Formosa' parents. Crop Breeding and Applied

Biotechnology, Viçosa, v. 6, n. 1, p. 24-29, 2006 a.

MARIN, S. L. D.; PEREIRA, M. G.; AMARAL JR, A. T.; MARTELLETO, L. A. P.; IDE, C. D. Partial diallel to evaluate the combining ability for economically important traits of papaya. Scientia Agricola, Piracicaba, v. 63, n. 6, p. 540-546, 2006 b.

MARTINS, G. N.; SILVA, R. F.; ARAÚJO, E. F.; PEREIRA, M. G.; VIEIRA, H. D.; VIANA, A. P. Influência do tipo de fruto, peso específico das sementes e períodos de armazenamento na qualidade fisiológica de sementes de mamão do grupo formosa. Revista Brasileira de Sementes, Londrina, v. 27, n. 2, p. 12-17, 2005.

MELETTI, L. M. M; SAMPAIO, A. C.; RUGGIERO, C. Avanços na fruticultura tropical no brasil. Revista Brasileira de Fruticultura, Jaboticabal, v. especial, p. 73-75, 2011.

NASCIMENTO, W. M.; PEREIRA, R. Testes para avaliação do potencial fisiológico de sementes de alface e sua relação com a germinação sob temperaturas adversas. Revista Brasileira de Sementes, Londrina, v. 29, $\mathrm{n}$. 3, p.175-179, 2007.

SANTOS, S. A.; SILVA, R. F.; PEREIRA, M. G.; ALVES, E. MACHADO, J. C.; BORÉM, F. M.; GUIMARÃES, R. M.; MARQUES, E. R. Estudos morfo-anatômicos de sementes de dois genótipos de mamão (Carica papaya L.). Revista Brasileira de Sementes, Londrina, v. 31, n. 2, p. 116-122, 2009.

SARI, M.; MURNIATI, E.; SUHARTANTO, M. R. Pengaruh sarcotesta dan pengeringan benih serta perlakuan pendahuluan terhadap viabilitas dan dormansi benih pepaya (Carica papaya L.). Jurnal Agronomi Indonesia, Bogor, v. 3, n. 2, p. 23-30, 2005.

SERRANO, L. A. L.; CATTANEO, L. F. O cultivo do mamoeiro no Brasil. Revista Brasileira de Fruticultura, Jaboticabal, v. 32, n. 3, 2010.

SINGH, D. The relative importance of characters affecting genetic divergence. The Indian Journal of Genetic and Plant Breeding, New Dehli, v. 36, p. 237-245, 1981. 
TOKUHISA, D.; SANTOS DIAS, D. C. F.; ALVARENGA, E. M.; SANTOS DIAS, L. A.; MARIN, S. L. D. Tratamentos para superação da dormência em sementes de mamão. Revista Brasileira de Sementes, Londrina, v. 29, n. 1, p. 131-139, 2007.

TOKUHISA, D.; DIAS, D. C. F. S.; ALVARENGA, E. M.; DIAS, L. A. S.; MARIN, S. L. D. Época de colheita dos frutos e ocorrência de dormência em sementes de mamão (Carica papaya L.). Revista Brasileira de Sementes, Londrina, v. 30, n. 2, p. 75-80, 2008.

VANZOLINI, S.; ARAKI, C. A. S.; SILVA, A. C. T. M.; NAKAGAWA, J. Teste de comprimento de plântula na avaliação da qualidade fisiológica de sementes de soja. Revista Brasileira de Sementes, Londrina, v. 29, n. 2, p. 90-96, 2007.

VENANCIO, L. P.; LOPES, J. C.; MACIEL, K. S.; COLA, M. P. A. Teste do envelhecimento acelerado para avaliação da qualidade fisiológica de sementes de milho. Enciclopédia Biosfera, Goiânia, v. 8, n. 14, p. 899906, 2012. 